\title{
Multi-scale Modelling of the Two-Dimensional Flow Dynamics in a Stationary Supersonic Hot Gas Expansion
}

\author{
Giannandrea Abbate ${ }^{1}$, Barend J. Thijsse ${ }^{2}$, and Chris R. Kleijn ${ }^{1}$ \\ ${ }^{1}$ Dept. of Multi-Scale Physics \& J.M.Burgers Centre for Fluid Mechanics, Delft \\ University of Technology, \\ Prins Bernhardlaan 6, Delft, The Netherlands \\ G.Abbate@klft.tn.tudelft.nl, C.R.kleijn@tudelft.nl, \\ http://www.msp.tudelft.nl \\ ${ }^{2}$ Dept. of Materials Science and Engineering, Delft University of Technology, \\ Mekelweg 2, Delft, The Netherlands \\ B.J.Thijsse@tnw.tudelft.nl, \\ http://www.3me.tudelft.nl
}

\begin{abstract}
A stationary hot gas jet supersonically expanding into a low pressure environment is studied through multi-scale numerical simulations.

A hybrid continuum-molecular approach is used to model the flow. Due to the low pressure and high thermodynamic gradients, the accuracy of continuum mechanics results are doubtful, while, because of its excessive time expenses, a full molecular method is not feasible. The results of the proposed hybrid continuum-molecular approach have been successfully validated against experimental data.

An important question for the full understanding of the processes governing the flow is addressed: the demonstration of an invasion of the supersonic part of the flow by background particles. Through the tracking of particles and collisions in the supersonic region it could be definitively proven that background particles are present in this region. We present a complete two dimensional picture of how the invading background particles distribute and collide with local particles into the supersonic region.
\end{abstract}

Keywords: Direct Simulation Monte Carlo, Coupled Method, Hybrid Method, Rarefied Gas Flow, Supersonic Expansion.

\section{Introduction}

The necessity to model a hot gas jet supersonically expanding in a low pressure environment is present in several gas fluidic applications of current importance; gas thruster nozzles and plume flows [1 and processes of thin film deposition from expanding plasma or gas jets 2] are some examples.

The gas in the jet is generally at relatively high pressure, and then it rapidly expands into a low pressure environment. For this reason, the gas first supersonically expands and then quickly compresses through a stationary shock wave 
(Mach disk). In addition, the expansion zone is surrounded by a barrel shaped shock (barrel shock) (Fig. 1).

Although several studies have been devoted to supersonic expansion of gas jets in a low pressure environment [3456], full understanding of the processes governing the flow has not been reached yet. In particular, it is still not completely clear whether, due to rarefaction effects, the barrel shock still protects the supersonic part of the flow from the invasion of background molecules, i.e. molecules that are present outside the expansion-shock region [3]. Invading the supersonic part of the jet, these background molecules could, therefore, influence its properties. Already Fenn and Anderson in 1966 [7] and Campargue in 1970 8] predicted this phenomenon, but a full understanding of it has not yet been reached.

In the current paper the important issue of the presence of background particles in the supersonic region will be addressed. This issue will be studied through the tracking of particles and collisions in the supersonic region.

It has been shown [5] that, because of rarefaction effects, the Navier-Stokes equations are not adequate for the description of this problem, and therefore, the continuum CFD (Computational Fluid Dynamics) approach fails in predicting the flow field in the expansion-shock region. A kinetic simulation model should be applied to correctly study the flow. For this reason, in the past, DSMC (Direct Simulation Monte Carlo) has been used [5]. However, because DSMC computational expenses scale with $\mathrm{Kn}^{-4}$, it was impossible to fulfill the DSMC requirements with respect to the size of gridcells and timesteps, expecially near the inlet, where the Knudsen number is quite low [5].

In order to overcome this problem, one needs to construct a model that on the one hand accounts for the molecular nature of the gas flow where needed, and on the other hand uses a continuum model where allowed. In the past years several hybrid continuum/molecular models have been proposed 91011121314.

The present hybrid method has been presented in [1213] and validated in [14. The method applies the continuum approach in the wide continuum region in order to save computational time and to get numerical simulations of the flow, pressure and temperature of the expanding jet at large scales. DSMC is only applied in the expansion-shock region where it is necessary in order to correctly model the rarefied nature of the flow and to show how the invading background particles distribute and collide with local particles into the supersonic region.

Whereas in [14] we focused on the validation of 1-dimensional temperature and velocity profiles along the axis against experimental data from [3], in the present paper we will present 2-dimensional velocity fields which will be compared to experimental data from [6].

\section{Studied Configuration and Measurement Technique}

In order to have an experimental support to our conclusions, all our numerical results have been validated by comparing them to experimental measurements by Engeln et al. 3], Vankan et al. [4] and Gabriel et al. 6]. 
The measurements described in [346] were performed on an expanding thermal plasma jet. Nevertheless, because of the low ionization degree, they can be used to validate our present results on a neutral gas flow, neglecting the presence of electrons and ions [3] and the effects of ionization and recombination on the flow field 3 15].

The experimental set-up in which the expanding thermal plasma jet is created has been extensively described in 16 and it is schematically depicted in Fig. 1

Two techniques, the Thomson-Rayleigh and the laser induced fluorescence spectroscopy (LIF), have been used to study the flow. The latter provided detailed, 2-dimensional information on the velocity field. A description of the two techniques is given in 34 .

\section{$3 \quad$ Numerical Simulation Method}

The present method has been described in detail in 1213. and validated in [14], here only a short description will be given.

In order to properly characterize the various regimes in the gas flow, the Knudsen number $K n$ is defined as the ratio between the mean free path and a relevant macroscopic length scale. When Knudsen is low $(K n<0.01)$, the gas may be treated as a continuum and the gas flow modelled using CFD (Computational Fluid Dynamics). When Knudsen is high $(K n>10)$, the gas behavior is molecular and may be modelled using Molecular Dynamics techniques. In the intermediate regime, the DSMC (Direct Simulation Monte Carlo) approach is the most commonly used simulation technique. However, its computational expenses scale with $K n^{-4}$ and it becomes very time demanding as $K n$ becomes lower than $\sim 0.05$.

In order to overcome this dilemma and solve the flow throughout the expanding gas jet, we use a hybrid CFD/DSMC model, which takes into account the molecular nature of the gas flow where needed, and uses a continuum model where allowed.

The continuum breakdown parameter $K n_{\max }[18$ is employed in the present study as a criterion for selecting the proper solver. If the calculated value of the continuum breakdown parameter in a region is larger than a limiting value $K n_{\text {split }}$, then that region cannot be accurately modelled using the N-S equations, and DSMC has to be used. For $K n_{\text {split }}$ a value of 0.05 was used. The method has been found to be rather insensitive to the precise CFD/DSMC interface location w.r.t. $K n_{\text {split }}[12$.

The CFD code used is a 2-D, unsteady code based on a finite volume formulation in compressible form. It uses an explicit, second-order, flux-splitting, MUSCL scheme for the Navier-Stokes equations.

The 2-D DSMC code developed is based on the algorithm described in [17]. A "particle reservoirs" approach was used to implement the inlet (outlet) boundary conditions. Molecules were generated in those reservoirs with a ChapmannEnskog velocity distributions. 


\subsection{Schwarz Coupling}

The multi-scale hybrid coupling approach used is based on the overlapped Schwarz method with Dirichlet-Dirichlet boundary conditions [10] and it consists of two stages.

The first stage is a prediction stage, where the unsteady N-S equations are integrated in time until steady state on the entire domain. From this steady state solution, the continuum breakdown parameter $K n_{\max }$ is computed and its values are used to split the domain in the subdomains, where the flow field will be evaluated respectively using DSMC and the N-S solver. Between the DSMC and CFD regions an overlap region is considered, where the flow is computed with both the DSMC and the CFD solver.

In the second stage, DSMC and CFD are run in their respective subdomains with their own time steps until steady state. The boundary conditions to the DSMC region come from the solution in the CFD region and are imposed by a "particle reservoir" approach, whereas the boundary conditions to the CFD region come from the solution in the DSMC region, averaged over the CFD cells.

Once a steady state solution has been obtained in both the DSMC and N-S regions, the continuum breakdown parameter $K n_{\max }$ is re-evaluated and a new boundary between the two regions is computed. This second stage is iterated until the relative difference (in pressure, velocity and temperature) between the DSMC and CFD solutions in the overlapping region is less than a prescribed value.

The advantage of using a Schwarz method with Dirichlet-Dirichlet boundary conditions, instead of the more common flux-based coupling technique [1], is that, the latter requires a much higher number of samples than the Schwarz method 11. In fact, the DSMC statistical scatter involved in determining the fluxes is much higher than that associated with the macroscopic state variables.

\subsection{Modelled Geometry}

The computational domain (Fig. 1) is a $d=32 \mathrm{~cm}$ diameter cylinder of length $L=50 \mathrm{~cm}$. From a circular hole of diameter $d_{i n}=8 \mathrm{~mm}$, on its top, a flow of $56 \mathrm{sccs}$ of argon is injected at a temperature $T_{i n}=8000 \mathrm{~K}$. The top and lateral walls are at a temperature $T_{w}=400 \mathrm{~K}$, while the bottom wall is at a temperature $T_{s u b}=600 \mathrm{~K}$. The pumping exit, which in reality is a circular hole, in our 2-D model has been represented as a $l_{\text {out }}=2 \mathrm{~cm}$ wide ring on the bottom of the cylinder at a distance of $R_{\text {out }}=12 \mathrm{~cm}$ from the axis. A pressure $P_{\text {out }}=20$ $P a$ in the exit has been considered, since for this outlet pressure a large amount of experimental data is available 36 .

Inside the chamber we suppose the flow to be 2-D axi-symmetric. The continuum grid is composed of 100 cells in the radial direction and 200 cells in the axial direction. Grid independence has been tested by doubling the continuum grid in each of the two directions, leading to variations in the solution below $3 \%$. The code automatically refines the mesh in the DSMC region to fullfill its requirements. The number of simulated particle in the DSMC region is $N \approx 8 \cdot 10^{5}$. 


\section{Diagram not to scale}

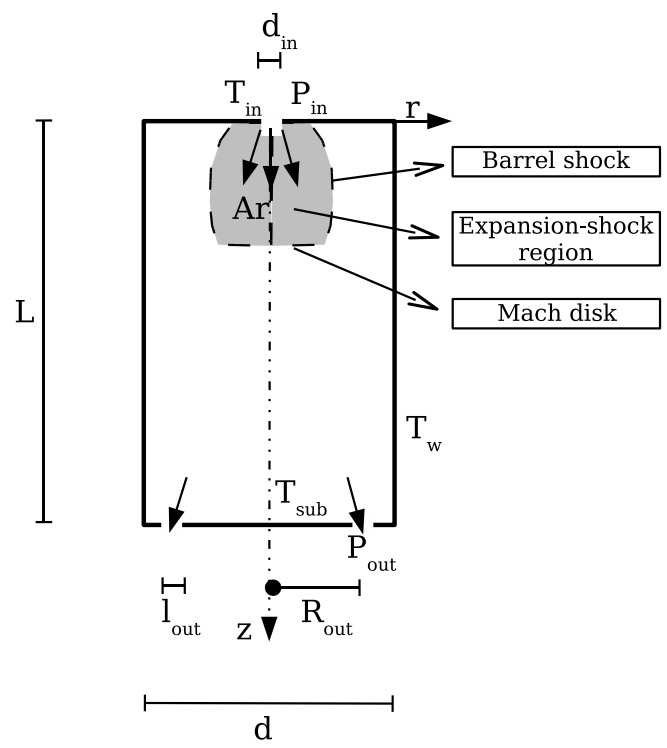

Fig. 1. Scheme of the low pressure chamber

\section{Results}

\subsection{General Flow Field Characteristics}

In Fig. 2 the number density (a) and pressure (b) profiles along the z-axis in the expansion-shock region, as evaluated by the hybrid approach at $20 \mathrm{~Pa}$ chamber pressure, are shown. It is well known [19] that, in the expansion, the density decreases quadratically with the distance $z$ to the inlet $\left(1 / z^{2}\right)$, whereas the pressure has a $1 / z^{2 \gamma}$ dependence, where $\gamma$ is the gas constant (for argon $\gamma=$ 1.67). In Fig. 2(a), the number density profiles measured with the ThomsonRayleigh technique by Vankan et al. at 40 and $10 P a$ chamber pressures are also presented. Even if no density measurements were available at $20 \mathrm{~Pa}$ chamber pressure, the present hybrid results are exactly between the experimental data at 40 and $10 P a$ chamber pressures measured by Vankan et al. 44.

Fig. 3(a) shows the division between the DSMC, continuum and overlapping regions in our hybrid method. In Fig. 3(b)-(e) a comparison between experimental data from [6], results from the present hybrid method, results from full DSMC simulations performed by Selezneva et al. [5], and results from present continuum simulations for the 2-dimensional velocity field in the expansion-shock region is presented. The velocity contours in Fig. 3(b) are the result of an interpolation of measured velocities at various positions in the expansion-shock region [6. It is clear that the hybrid method predicts the experimental data better than the 

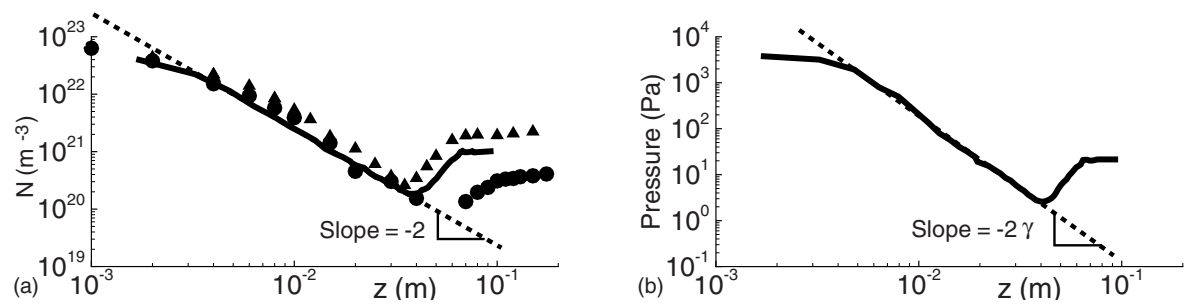

Fig. 2. Number density (a) and pressure (b) distributions along z-axis in the expansionshock region. Hybrid approach at $20 \mathrm{~Pa}$ (solid kernel), Theoretical trend in the expansion (dashed kernel), Experimental number density distribution from [4] at $10 \mathrm{~Pa}$ (bullet), and at $40 \mathrm{~Pa}$ (triangle).

other approaches. The reason why the hybrid approach predicts experimental data even better than the full DSMC simulations is that, as discussed in section 1 and as already highlighted by Selezneva et al. [5], in the full DSMC simulations it was not possible to respect DSMC requirements in the near inlet region and a too coarse mesh had to be used.

If we first compare the experimental data from [6] (Fig. 3(b)) to the results of the full CFD approach (Fig. 3(e)), the velocity predicted by the continuum approach in the expansion-shock region is significantly $(200-500 \mathrm{~m} / \mathrm{s})$ lower than the experimental one. Because of rarefaction, in fact, upstream of the shock the expansion is stronger, reaching higher velocity values.

If we compare the experimental data from [6] (Fig. [3(b)) to the full DSMC simulations by Selezneva et al. [5] (Fig. 3](d)), we can notice that the DSMC predicts correct velocity values in the expansion, but the maximum velocity along the z-axis is moved $\sim 1 \mathrm{~cm}$ upstream with respect to the experimental data.

Finally, hybrid simulations (Fig. 3(c)) result in a very good agreement with the experiments (Fig. 3(b)); The hybrid approach, in fact, was able to predict the correct velocity values and the right position for the velocity peak in the expansion.

\subsection{Invasion of the Expansion-Shock Region}

In this section we want to demonstrate the invasion of background particles into the expansion-shock region. In continuum conditions, because of the presence of the shock, these particles would not be able to enter the supersonic region. However, we will show that, because of the rarefaction effects, the shock becomes transparent and does not protect the supersonic region. Therefore some particles may actually move into it from the subsonic part of the flow. To demonstrate this hypothesis, it is necessary to know the origin of the particles present in the supersonic region. For this reason, for the particles in the DSMC region, two different labels were used; one for the particles which, after entering the reactor chamber, have always been in the supersonic region (the so called "inlet particles"), and a different one for the background particles. 

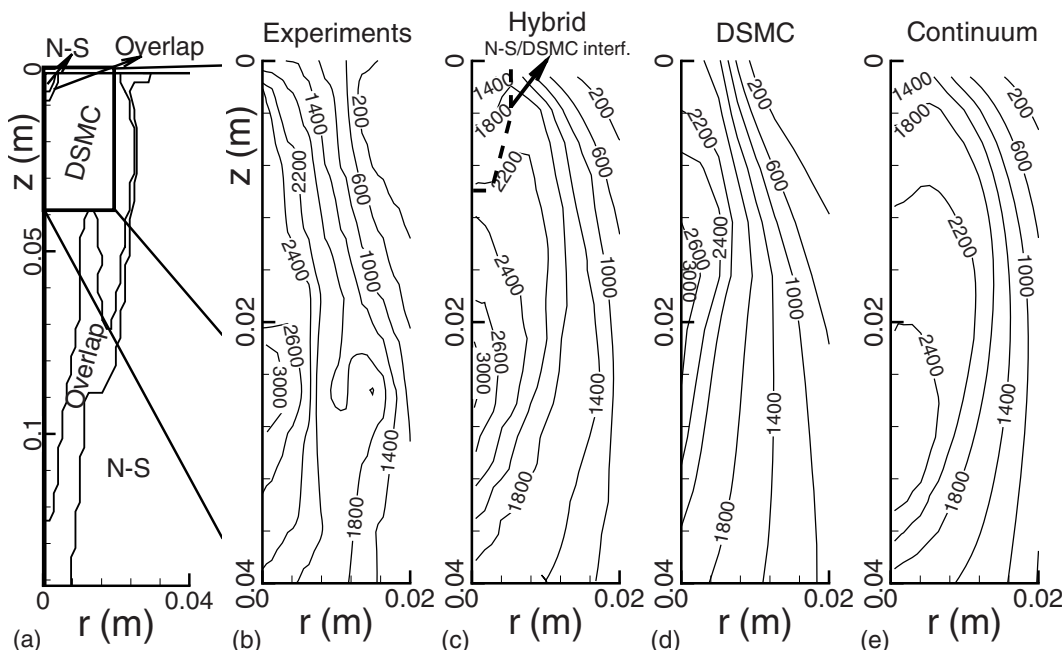

Fig. 3. N-S/DSMC domains splitting (a) and velocity field $(\mathrm{m} / \mathrm{s}$ ) zoomed in the expansion-shock region at $20 \mathrm{~Pa}$ chamber pressure. Experimental data from [6] (b), present hybrid simulations (c), DSMC data from [5] (d), present continuum simulations (e).

In Fig. 4 we compare the axial velocity distribution of our simulation at $r=0$ and $z=59 \mathrm{~mm}$ (a) and the radial velocity distribution of our simulation at $r=22 \mathrm{~mm}$ and $z=50 \mathrm{~mm}$ (b) to the ones measured by Engeln et al. It is clear that there is a very good agreement between our current hybrid simulations and the experiments from 3 .

In Fig. 4 we also show the contribution of the background particles and the inlet particles to the axial velocity distribution, at the position $r=0$ and $z=59$ $m m$ (a), and to the radial velocity distribution at the position $r=22 \mathrm{~mm}$ and $z=50 \mathrm{~mm}$ (b). The presence of background particles in the supersonic region is evident. The peaks of the two contributions to the radial velocity distribution (Fig. 4(b)) are located on opposite sides of the zero velocity position, meaning that particles coming from the inlet are moving away from the axis because of the expansion, whereas background particles are penetrating into the supersonic region and moving toward the axis. Once the background particles have penetrated the supersonic region, they start colliding and interacting with the particles that are already there, decelerating them and being accelerated by them.

In order to further prove the hypothesis of the presence of background particles in the supersonic region and explain how they collide and interact with the local particles, a study was performed, at the molecular scale, by tracking particles and collisions in the supersonic region. The results of this study are presented in Fig. 5.

The background particles concentration in Fig. [5(a) further proves their presence in the supersonic region. 

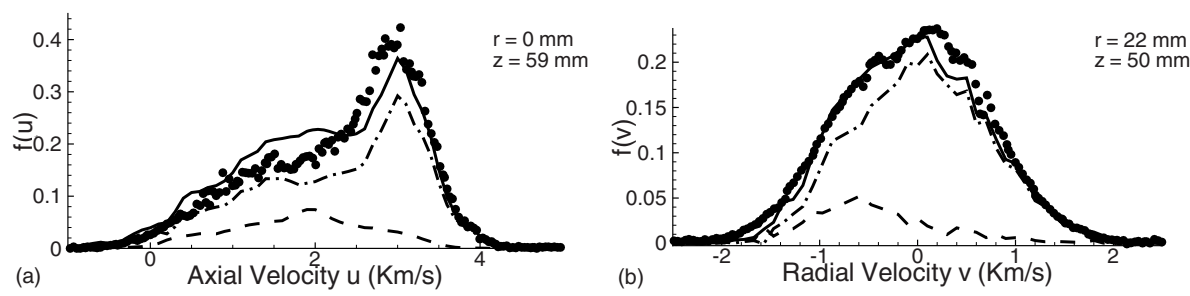

Fig. 4. Relative contribution of background particles to the axial velocity distribution at $r=0$ and $z=59 \mathrm{~mm}$ (a) and to the radial velocity distribution at $r=22 \mathrm{~mm}$ and $z=50 \mathrm{~mm}$ (b). Hybrid simulation total velocity distribution (solid kernel), experimental total velocity distribution from [3] (bullet), Inlet particles contribution (dash-dotted kernel), Background particles contribution (dashed kernel).
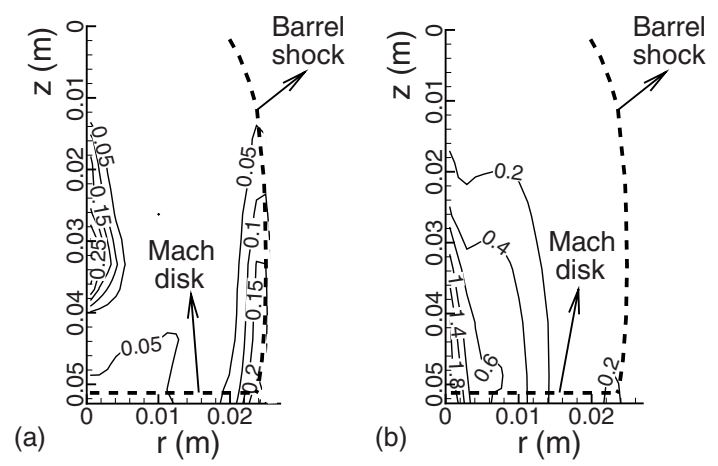

Fig. 5. Fractional concentration of background particles in the supersonic region (a) and average number of times that inlet particles have collided with background particles before reaching the given location (b).

In the expansion region, the velocity increases, reaching a maximum value on the axis at a distance $z=3 \mathrm{~cm}$ from the inlet (Fig. 3), whereas density and pressure decrease reaching a minimum (Fig. 2) at the same location. The invading background particles are driven into the region of minimum pressure by favorable pressure gradients. For the same reason, once they are there, it is difficult for them to cross the Mach disk because of the adverse pressure gradient. Therefore, the invading background particles concentrate in the region of minimum pressure, reaching values of up $25 \%$ of the total number of particles.

Finally, Fig. 5(b) presents the average number of collisions with background particles that an inlet particle has undergone before reaching its position. As expected, the number of collisions increases along the $\mathrm{z}$ axis and it reaches the maximum value of $\sim 1.8$ collisions. This is of course an averaged value, meaning that there are inlet particles which did not collide as well as inlet particles that have collided much more than 1.8 times with background particles. This clearly 
demonstrates that the inlet particles do interact with the background particles that invaded the supersonic region.

Engeln et al. in [3] and Gabriel et al. in 6] also found experimental indications for the presence of background particles in the expansion-shock region. Therefore, our study gives a numerical support to the hypothesis of Engeln et al. and Gabriel et al. that background particles can penetrate the supersonic region and, by interacting with the inlet particles, can influence the flow field.

\section{Conclusions}

The gas dynamics of a hot gas jet supersonically expanding into a low pressure environment is studied by means of a multi-scale hybrid coupled continuumDSMC method. This method gives the possibility to save computational time using CFD in most of the domain and to use DSMC only where it is necessary in order to correctly model the flow.

The answer to an important question about supersonic expansion in a low pressure environment has been found: the invasion of the supersonic region by background particles. By tracking particles and collisions in the supersonic region, we have demonstrated the presence of background particles in this region, thus proving the invasion of the supersonic region by background particles and describing how they can influence the flow field by colliding and interacting with the local particles.

Acknowledgments. We thank Profs. D.C.Schram, M.C.M.Van de Sanden, R.Engeln and O.Gabriel for usefull discussions and for making available to us their experimental data and the DCSE (Delft Centre for Computational Science and Engineering) for financial support.

\section{References}

1. Cai, C., Boyd, I.D.: 3D Simulation of Plume Flows from a Cluster of Plasma Thrusters. In: $36^{\text {th }}$ AIAA Plasmadynamics and Laser Conference, Toronto, Ontario, Canada, June 6-9, 2005, AIAA-2005-4662 (2005)

2. Gielen, J.W.A.M., Kessels, W.M.M., van de Sanden, M.C.M., Schram, D.C.: Effect of Substrate Conditions on the Plasma Beam Deposition of Amorphous Hydrogenated Carbon. J. Appl. Phys. 82, 2643 (1997)

3. Engeln, R., Mazouffre, S., Vankan, P., Schram, D.C., Sadeghi, N.: Flow Dynamics and Invasion by Background Gas of a Supersonically Expanding Thermal Plasma. Plasma Sources Sci. Technol. 10, 595 (2001)

4. Vankan, P., Mazouffre, S., Engeln, R., Schram, D.C.: Inflow and Shock Formation in Supersonic, Rarefied Plasma Expansions. Phys. Plasmas 12, 102-303 (2005)

5. Selezneva, S.E., Boulos, M.I., van de Sanden, M.C.M., Engeln, R., Schram, D.C.: Stationary Supersonic Plasma Expansion: Continuum Fluid Mechanics Versus Direct Simulation Monte Carlo Method. J. Phys. D: Appl. Phys. 35, 1362 (2002) 
6. Gabriel, O., Colsters, P., Engeln, R., Schram, D.C.: Invasion of Molecules and Supersonic Plasma Expansion. In: Proc. 25th Int. Symph. Rarefied Gas Dynamics, S.Petersburg, Russia (2006)

7. Fenn, J.B., Anderson, J.B.: Rarefied Gas Dynamics. In: de Leeuw, J.H. (ed.), 2nd edn. Academic Press, New York (1966)

8. Campargue, R.: Aerodynamic Separation Effect on Gas and Isotope Mixtures Induced by Invasion of the Free Jet Shock Wave Structure. J. Chem. Phys. 52, 1795 (1970)

9. Le Tallec, P., Mallinger, F.: Coupling Boltzmann and Navier-Stokes Equations by Half Fluxes. Journal Computational Physics 136, 51 (1997)

10. Wu, J.S., Lian, Y.Y., Cheng., G., Koomullil, R.P., Tseng, K.C.: Development and Verification of a Coupled DSMC-NS Scheme Using Unstructured Mesh. Journal of Computational Physics 219, 579 (2006)

11. Schwartzentruber, T.E., Boyd, I.D.: A Hybrid Particle-Continuum Method Applied to Shock Waves. Journal of Computational Physics 215(2), 402 (2006)

12. Abbate, G., Thijsse, B.J., Kleijn, C.R.: An Adaptive Hybrid Navier-Stokes/DSMC Method for Transient and Steady-State Rarefied Gas Flows Simulations. Journal Computational Physics (submitted)

13. Abbate, G., Thijsse, B.J., Kleijn, C.R.: Coupled Navier-Stokes/DSMC Method for Transient and Steady-State Gas Flows. In: Shi, Y., van Albada, G.D., Dongarra, J., Sloot, P.M.A. (eds.) ICCS 2007. LNCS, vol. 4487, p. 842. Springer, Heidelberg (2007)

14. Abbate, G., Thijsse, B.J., Kleijn, C.R.: Validation of a Hybrid NavierStokes/DSMC Method for Multiscale Transient and Steady-State Gas Flows. special SMMS 2007 issue of International Journal Multiscale Computational Engineering 6(1), 1 (2008)

15. Selezneva, S.E., Rajabian, M., Gravelle, D., Boulos, M.I.: Study of the Structure and Deviation From Equilibrium in Direct Current Supersonic Plasma Jets. J. Phys. D: Appl. Phys. 34(18), 2862 (2001)

16. van de Sanden, M.C.M., de Regt, J.M., Jansen, G.M., van der Mullen, J.A.M., Schram, D.C., van der Sijde, B.: A Combined Thomson-Rayleigh Scattering Diagnostic Using an Intensified Photodiode Array. Rev. Sci. Instrum. 63, 3369 (1992)

17. Bird, G.A.: Molecular Gas Dynamics and Direct Simulation Monte Carlo. Claredon Press Oxford Science (1998)

18. Wang, W.L., Boyd, I.D.: Continuum Breakdown in Hypersonic Viscous Flows. In: 40th AIAA Aerospace Sciences Meeting and Exhibit, January 14-17, 2002, Reno, NV (2002)

19. Ashkenas, H., Sherman, F.S.: Experimental Methods in Rarefied Gas Dynamics. In: de Leeuw, J.H. (ed.) Rarefied Gas Dynamics, vol. II, p. 84. Academic Press, New York (1965) 